\title{
INTERNATIONAL CENTRE FOR THEORETICAL PHYSICS
}

\author{
SCHWINGER-BOSON STUDIES \\ OF THE SINGLE HOLE MOTION \\ IN A 2d QUANTUM ANTIFERROMAGNET
}

C.Q. Wu

Z.B. Su

and

L. Yu
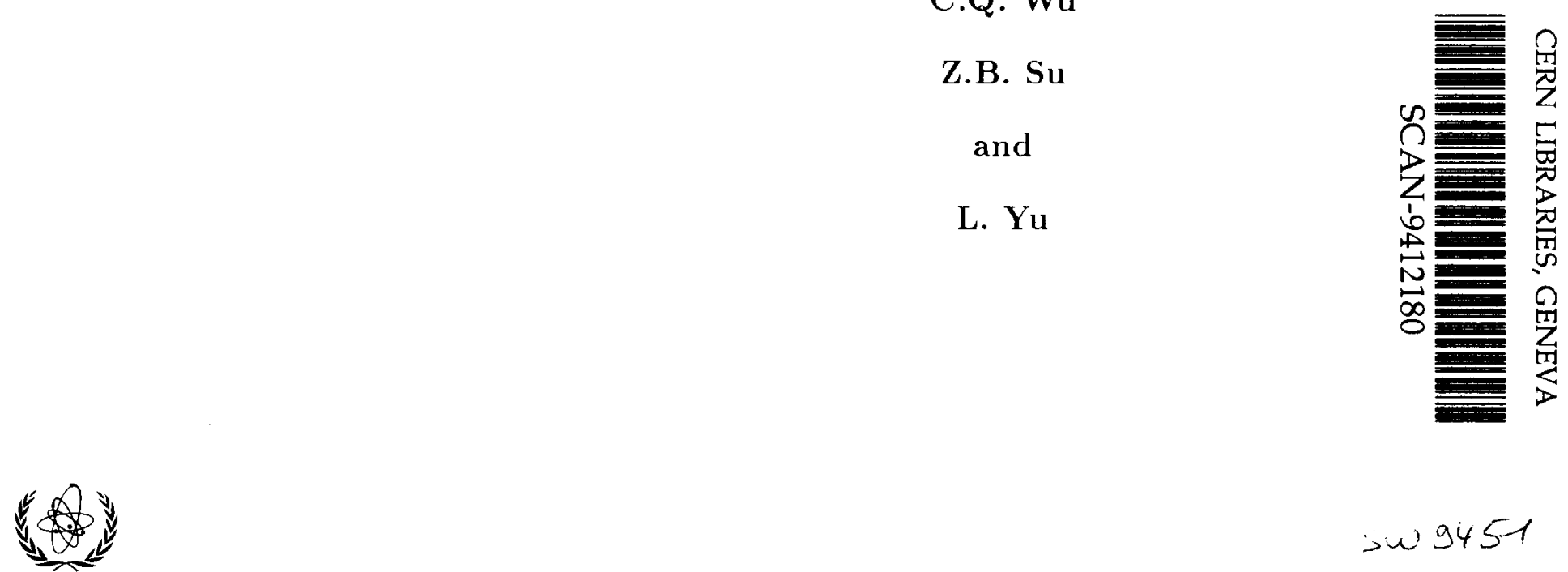

309451

INTERNATIONAL ATOMIC ENERGY

AGENCY

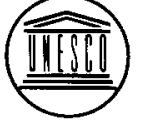

UNITED NATIONS EDUCATIONAL, SCIENTIFIC AND CULTURAL ORGANIZATION 
International Atomic Energy Agency

and

United Nations Educational Scientific and Cultural Organization

INTERNATIONAL CENTRE FOR THEORETICAL PHYSICS

\title{
SCHWINGER-BOSON STUDIES OF THE SINGLE HOLE MOTION IN A 2d QUANTUM ANTIFERROMAGNET
}

\author{
C.Q. Wu \\ International Centre for Theoretical Physics, Trieste, Italy \\ and \\ T.D. Lee Physics Laboratory and Department of Physics, Fudan University, \\ Shanghai 200433, People's Republic of China, \\ Z.B. $\mathrm{Su}$ \\ Institute of Theoretical Physics, Chinese Academy of Science, \\ Beijing 100080, People's Republic of China \\ and \\ L. $\mathrm{Yu}$ \\ International Centre for Theoretical Physics, Trieste, Italy \\ and \\ Institute of Theoretical Physics, Chinese Academy of Science, \\ Beijing 100080, Peole's Republic of China.
}

\begin{abstract}
Within the Schwinger-boson approach for the $t-J$ model, the single hole problem in a two-dimensional quantum antiferromagnet is studied by using the quantum Bogoliubovde Gennes formalism which treats the distortion of the spin background and quantum spin fluctuations on an equal footing. Several self-trapped localized hole states are found in the distorted spin-background as in the case of an anisotropic Heisenberg model. These localized hole states survive at finite temperatures when the antiferromagnetic order becomes short-ranged. The energy separation between the two lowest states is reduced by considering the spin-background distortion, but it remains finite.
\end{abstract}

MIRAMARE - TRIESTE

September 1994 


\section{INTRODUCTION}

The discovery of the high $T_{c}$ superconductors [1] has given rise to great interest in twodimensional $(2 \mathrm{~d})$ strongly correlated electron systems since there is a consensus now that the $2 \mathrm{~d} \mathrm{CuO}_{2}$ plane is mainly responsible for the high transition temperature superconductivity of copper oxides and the electrons in the $\mathrm{CuO}_{2}$ plane are strongly correlated. Without doping, the $2 \mathrm{~d} \mathrm{CuO}$ plane is in an antiferromagnetic (AF) state at $T=0[2]$ and is well described by an isotropic spin- $\frac{1}{2}$ Heisenberg model. Upon doping the AF long range order fades away and superconductivity occurs below certain critical temperature. Whether the superconductivity is caused by magnetic fluctuations, is a separate issue. It is, however, crucial to understand the behaviour of charge carriers on an AF background.

In the early days of the high- $T_{c}$ heat wave Anderson [3] suggested that the basic physics of the high- $T_{c}$ superconductors should be that of a doped Mott insulator described by the one-band Hubbard model

$$
H=-\sum_{\langle i, j\rangle, \sigma} C_{i \sigma}^{\dagger} C_{j \sigma}+U \sum_{i} C_{i \uparrow}^{\dagger} C_{i \uparrow} C_{i \downarrow}^{\dagger} C_{i l}
$$

where the indices $i, j$ run over $N$ lattice sites, $\langle i, j\rangle$ indicates the summation over nearest neighbours, $C_{i \sigma}^{\dagger}\left(C_{i \sigma}\right)$ are the electron creation (annihilation) operators of spin $\sigma$ at site $i$, and the two-body interaction is on-site and repulsive $(U>0)$.

In the large $U$ limit, it is more convenient to use a canonical transformation to project out the doubly occupied sites costing energy $U$ and to obtain an equivalent, the so-called $t-J$ model [4]:

$$
H=-t \sum_{\langle i, j\rangle \sigma} C_{i \sigma}^{\dagger} C_{j \sigma}+J \sum_{\langle i, j\rangle} \mathbf{S}_{i} \cdot \mathbf{S}_{j},
$$

where the restriction to the Hilbert space without double occupacy is assumed and the superexchange interaction between the nearest neighbour sites $J=4 t^{2} / U$. The spin density operator is $\mathbf{S}_{i}=\frac{1}{2} C_{i}^{\dagger} \sigma C_{i}$, where $\sigma$ are the Pauli matrices. The $t-J$ model can be studied independently, even in the limit $t \ll J$, but the correspondence with the Hubbard model is established only in the limit $t \gg J$, or $U \gg t$. As shown by Zhang and Rice [5], the low-energy physics of the $\mathrm{CuO}_{2}$ plane is determined by the singlet state formed by the additional hole on oxygen with the existing hole on copper. The hopping of this singlet is described by an effective $t-J$ model which makes this model even more pertinent to high- $T_{c}$ superconductors.

The single hole motion on an AF background has been extensively studied by many authors. Most of the references are quoted in a recent review article [6]. In particular, this problem has been considered through an exact diagonalization using the Lanczos-type algorithms for clusters up to 26 sites [7], but the limitation to small clusters may give rise to artifacts due to finite sizes. Within a self-consistent Born approximation, Schmitt-Rink, Varma, and Ruckenstein [8], and Kane, Lee, and Read [9], considered the renormalization of the hole motion due to virtual excitations of spin waves using Green's function techniques. However, the distortion of the spin background caused by the presence of a hole is not included in their calculations. Considering a slightly generalized $t-J$ model with anisotropic exchange interactions, i.... 


$$
H=-t \sum_{\langle i, j\rangle \sigma} C_{i \sigma}^{\dagger} C_{j \sigma}+J \sum_{\langle i, j\rangle}\left[S_{i}^{z} S_{j}^{z}+\alpha\left(S_{i}^{x} S_{j}^{x}+S_{i}^{y} S_{j}^{y}\right)\right]
$$

$(\alpha \neq 1)$, the single hole problem has been carefully studied by two of us with collaborators earlier [10]. A new quantum Bogoliubov-de Gennes (BdeG) formalism has been successfully developed there to study the self-consistent motion of holes and spin excitations. The effects of local distortion of spin configurations and the renormalization of the hole motion due to virtual excitations of the distorted spin background are treated on an equal footing. Several localized hole states have been found in that study. Those states seem to survive upon extrapolation to the isotropic case. However, this has to be checked independently because the spin wave spectrum is quite different for the isotropic and the anisotropic cases. The gap of spin excitations vanishes only at $\alpha=1$. On the other hand, we know that the existence of self-trapped states, at least for the phonon case, depends strongly on the dimensionality of the system and the property of the excitation spectrum (gapless or gapful) [11]. Since the $\mathrm{CuO}_{2}$ plane of the high- $T_{c}$ superconductors is basically isotropic, we are more interested in the single hole problem in the isotropic $t-J$ model. An important question is whether the localized hole states found in the anisotropic $t-J$ model (3) survive in the isotropic $t-J$ model (2). Another equally important issue is whether these states still exist at finite temperatures when the AF order becomes short-ranged.

In this paper, we follow Ref. [10] to study the single hole problem in an AF background within the $t-J$ model (2) using the quantum BdeG formalism. We employ the Schwingerboson approach $[12,13]$, which should be suitable to the study of the single hole problem since, in the hole-free case, the mean-field (MF) approximation in this approach gives a quite good description of the AF background satisfying the Marshall sign rule. At zero temperature, the Schwinger bosons condense and the MF ground state is of a long range AF order. At finite temperatures, the AF order becomes short ranged. Moreover, the formalism itself is $S U(2)$ rotational invariant. Therefore, it is an ideal tool to explore the above mentioned open questions in the previous study [10]. In fact the answer to both questions is positive, namely, the self-trapped states exist for the isotropic Heisenberg model and they survive at finite temperatures.

The rest of the paper is organized as follows. We first consider the $t-J$ model within the Schwinger boson approach in Sec. II. Then the formalism for determining self-consistently the hole states is presented in Sec. III. Finally, in Sec. IV we present and discuss our results.

\section{II. t-J MODEL IN SCHWINGER-BOSON SCHEME}

Within the Schwinger-boson scherne [12,13], the electron operator $C_{i \sigma}$ can be expressed as a product of the Schwinger-boson operator $b_{i \sigma}$ and a slave-fermion (holon) operator $e_{i}$, i.e., $C_{i \sigma}^{\dagger}=e_{i} b_{i \sigma}^{\dagger}$, to implement the single occupancy constraint in the Hilbert space for the $t-J$ model. The $t-J$ Hamiltonian (2) then reads as:

$$
H=-t \sum_{\langle i j\rangle \sigma} e_{i}^{\dagger} e_{j} b_{j \sigma}^{\dagger} b_{i \sigma}-\frac{J}{2} \sum_{\langle i j\rangle}\left(b_{i \uparrow}^{\dagger} b_{j \downarrow}^{\dagger}-b_{i \downarrow}^{\dagger} b_{j \uparrow}^{\dagger}\right)\left(b_{i \uparrow} b_{j \downarrow}-b_{i \downarrow} b_{j \uparrow}\right)
$$

To describe the AF order and the ferromagnetic (FM) order, we introduce two order parameters 


$$
\Delta_{i j}=\left\langle b_{i \uparrow}^{\dagger} b_{j \downarrow}^{\dagger}-b_{i \downarrow}^{\dagger} b_{j \uparrow}^{\dagger}\right\rangle, \quad \chi_{i j}=\left\langle b_{i \uparrow}^{\dagger} b_{j \uparrow}+b_{i \downarrow}^{\dagger} b_{j \downarrow}\right\rangle,
$$

where $i$ runs over sublattice $\mathrm{A}$, while its nearest neighbour $j=i+\alpha(\alpha=\hat{x}, \hat{y},-\hat{x},-\hat{y})$, is on sublattice $B$.

In the hole-free case, the $t-J$ model reduces to the Heisenberg Hamiltonian. The ground state should have a uniform AF order parameter $\Delta$ and a vanishing FM order parameter $\chi$ in the MF approximation. Then we have the MF hamiltonian for the Heisenberg AF phase,

$$
H_{0}=\frac{1}{2} N J+N J \Delta^{2}+\lambda \sum_{i \sigma} b_{i \sigma}^{\dagger} b_{i \sigma}-J \Delta \sum_{i \in A} \sum_{\alpha}\left(b_{i \uparrow} b_{i+\alpha \downarrow}-b_{i \downarrow} b_{i+\alpha \uparrow}+b_{i \uparrow}^{\dagger} b_{i+\alpha \downarrow}^{\dagger}-b_{i \downarrow}^{\dagger} b_{i+\alpha \uparrow}^{\dagger}\right),
$$

where $\lambda=\mu-J$, and the chemical potential $\mu$ has been introduced to restrict the average occupancy to one. Carrying out the Fourier transformation $b_{i \sigma}=\frac{1}{\sqrt{N}} \sum_{k} e^{i \mathbf{k} \cdot \mathbf{A}}{ }_{i} b_{k \sigma}$, we have $H_{0}$ as

$$
H_{0}=\frac{1}{2} N J+N J \Delta^{2}+\lambda \sum_{k \sigma} b_{k \sigma}^{\dagger} b_{k \sigma}+J \sum_{k}\left(\gamma_{k} b_{k \uparrow} b_{-k+Q \downarrow}+\gamma_{k} b_{k \uparrow}^{\dagger} b_{-k+Q \downarrow}^{\dagger}\right),
$$

where $\gamma_{k}=\Delta\left(\cos k_{x}+\cos k_{y}\right)$ and $Q=( \pm \pi, \pm \pi)$. Now we can use the Bogoliubov transformation

$$
b_{k \uparrow}=u_{k} \cdot \alpha_{k}-v_{k} \beta_{k}^{\dagger}, \quad b_{-k+Q !}=u_{k} \beta_{k}-v_{k} \alpha_{k}^{\dagger},
$$

to diagonalize the hamiltonian $H_{0}$. We find

$$
u_{k}=\sqrt{\frac{\lambda+E_{k}}{2 E_{k}}} e^{i \theta_{k} / 2}, \quad v_{k}=\sqrt{\frac{\lambda-E_{k}}{2 E_{k}}} e^{i \theta_{k} / 2}
$$

where $\theta_{k}$ is defined by $\gamma_{k}=\left|\gamma_{k}\right| e^{i \theta_{k}}$, and the eigen-energy for both $\alpha_{k}$ and $\beta_{k}$ excitations is

$$
E_{k}=\sqrt{\lambda^{2}-\left|\gamma_{k}\right|^{2}}
$$

At finite temperatures. there is a gap $E_{g}=\sqrt{\lambda^{2}-(2 \Delta)^{2}}$ in the spin wave spectrum so that the Bogoliubov transformation can be performed over the entire Brillouin zone. Then we get the self-consistent equations for the order parameters

$$
1=\frac{1}{N} \sum_{k} \frac{\lambda}{E_{k}} \operatorname{coth} \frac{E_{k}}{2 T}, \quad \quad \quad \quad=\frac{1}{N} \sum_{k} \frac{\gamma_{k}\left(\cos k_{x}+\cos k_{y}\right)}{2 E_{k}} \operatorname{coth} \frac{E_{k}}{2 T} \text {. }
$$

At zero temperature, the spin-excitation gap vanishes and the Schwinger bosons condense at momentum $\mathbf{k}=\mathbf{K}_{ \pm}= \pm(\pi / 2, \pi / 2)$.

In the low temperature limit, the spin gap is much smaller than the temperature itself. Consider the density of spin-wave excitations, defined as

$$
\begin{aligned}
\zeta(E) & =\sum_{k} \delta\left(E-E_{k}\right)\left\langle\alpha_{k}^{\dagger} \alpha_{k}\right\rangle=\sum_{k} \delta\left(E-E_{k}\right)\left\langle\beta_{k}^{\dagger} \beta_{k}\right\rangle \\
& =\sum_{k} \frac{\delta\left(E-E_{k}\right)}{E^{E_{k} / T}-1}
\end{aligned}
$$


at a low, but finite temperature $T$, instead of the density of spin-wave states $\rho(E)=\sum_{k} \delta(E-$ $\left.E_{k}\right)$. Due to the fact that at low temperatures the energy gap of the spin-wave spectrum is much smaller than the temperature $T$, the boson occupancy of the spin wave states may be very high, although the density itself is very low at low energies. As a result of combining these two factors, i.e., the Bose distribution and density of states, the density of excitations shows a sharp maximum at low energies which is consistent with boson condensation at $T=0$. In Fig. 1 we show these two quantities at a finite temperature $T / \lambda=0.38$, where the spin gap $E_{g} / \lambda=0.01$. The peak appearing near zero energy at a finite temperature $T$ should correspond to the boson condensation at $T=0$. This means that there is a continuous crossover from $T \neq 0$ to $T=0$ limit. The calculation of the spin-spin correlation functions shows [13] that there is a long-range AF order at zero temperature, and the correlation decays exponentially with a finite correlation length $\xi$ at a finite temperature. At $T / \lambda=0.38$, the correlation length $\xi \approx 25$.

\section{QUANTUM BOGOLIUBOV-DE GENNES FORMALISM}

For the motion of a single hole in an AF background, there are the following major effects: The quantum fluctuations of the spin systems, the renormalization effect due to virtual process of emitting and reabsorbing the spin excitations, and the local distortion of the spin background. The first two effects have been considered in the self-consistent Born approximation [8,9]. The quantum BdeG formalism proposed in Ref. [10] treats all these effects equally. In this Section, we formulate the basic quantum BdeG equations for the single hole problem in the Schwinger boson approach.

First, we introduce the Green's function for the hole propagation,

$$
i G\left(i, j ; t, t^{\prime}\right)=\left\langle e_{i}(t) e_{j}^{\dagger}\left(t^{\prime}\right)\right\rangle
$$

and then the Dyson equation for the retarded Green's functions can be written as

$$
G_{r}(\omega ; i, j)=G_{r}^{(0)}(\omega ; i, j)+\sum_{l, l^{\prime}} G_{r}^{(0)}(\omega ; i . l) \Sigma_{r}\left(\omega ; l, l^{\prime}\right) G_{r}\left(\omega ; l^{\prime}, j\right),
$$

where, $\Sigma_{r}\left(\omega ; l, l^{\prime}\right)$ is the retarded hole self-energy, and $G^{(0)}(\omega ; i, j)$ is the bare hole Green's
function.

Within the adiabatic approximation, the system we are considering can be viewed as a composite of a single hole and an accompanying distorted spin background, i.e., the corresponding state is a direct product of these two parts

$$
|\Psi\rangle=|\Psi\rangle_{h} \otimes|\Psi\rangle_{s}
$$

In principle, the adiabatic approximation is valid only for the case $t \ll J$, but it turned out to give quite reasonable results even for the case when $t$ and $J$ are comparable [10]. The retarded Green's function for the hole is then

$$
G_{r}(\omega ; i, j)=\sum_{\alpha} \frac{\psi_{\alpha}(i) \psi_{\alpha}^{*}(j)}{\omega-E_{\alpha}+i \eta}
$$


where $\psi_{\alpha}$ and $E_{\alpha}$ are $\alpha$-th eigenstate and its corresponding energy, respectively. In the absence of interactions between the hole and its background, the Green's function is

$$
G_{r}^{(0)}(\omega ; i, j)=\frac{\delta_{i j}}{\omega+i \eta}
$$

Substituting the expressions of $G_{r}(\omega ; i, j)$ and $G_{r}^{(0)}(\omega ; i, j)$ into the Dyson equation (14), we obtain the so-called BdeG equation [10]

$$
E_{n} \psi_{n}(i)=\sum_{j} \Sigma_{r}\left(E_{n} ; i, j\right) \psi_{n}(j)
$$

which determines self-consistently the excitation energies and the wave functions of the hole states.

The interaction between the hole and its background is mainly due to the hopping term of the $t-J$ Hamiltonian (4). Up to the second-order perturbation, the self-energy of the hole includes both Hartree and Fock terms, and the corresponding self-energy diagrams are shown in Fig. 2. The Hartree and Fock self-energies are given as follows

$$
\begin{aligned}
\Sigma^{\mathrm{H}}\left(l, l^{\prime} ; t, t^{\prime}\right)= & 2 t^{2} \delta\left(t-t^{\prime}\right) I_{l l^{\prime}} \sum_{\left(m m^{\prime}\right)} \int d t_{1} G\left(m, m^{\prime} ; t_{1}, t_{1}\right) \\
& {\left[g^{22}\left(l-m, t-t_{1}\right) g^{11}\left(l^{\prime}-m^{\prime}, t-t_{1}\right)\right.} \\
& \left.-g^{21}\left(l-m^{\prime}, t-t_{1}\right) g^{12}\left(l^{\prime}-m, t-t_{1}\right)\right], \\
\Sigma^{\mathrm{F}}\left(l, l^{\prime} ; t, t^{\prime}\right)= & 2 t^{2} \sum_{m, m^{\prime}} I_{l m} I_{l^{\prime} m^{\prime}}\left(r\left(m, m^{\prime}: t, t^{\prime}\right) .\right. \\
& {\left[g^{21}\left(l-m^{\prime}, t-t^{\prime}\right) g^{12}\left(m-l^{\prime}, t-t^{\prime}\right)\right.} \\
& \left.-g^{22}\left(l-l^{\prime}, t-t^{\prime}\right) g^{11}\left(m-m^{\prime}, t-t^{\prime}\right)\right],
\end{aligned}
$$

where, $I_{l l^{\prime}}$ equals one for $l, l^{\prime}$ being the nearest neighbour sites and zero for others, while the spin Green's functions are defined as

$$
\begin{aligned}
i g\left(l, l^{\prime} ; t, t^{\prime}\right) & =\left(\begin{array}{cc}
i g^{11}\left(l, l^{\prime} ; t, t^{\prime}\right) & i g^{12}\left(l, l^{\prime} ; t, t^{\prime}\right) \\
i g^{21}\left(l, l^{\prime}: t, t^{\prime}\right) & i g^{22}\left(l, l^{\prime} ; t, t^{\prime}\right)
\end{array}\right) \\
& =\left(\begin{array}{cc}
\left\langle b_{l \uparrow}(t) b_{l^{\prime} \uparrow}^{\dagger}\left(t^{\prime}\right)\right\rangle & \left\langle b_{l_{1}}(t) b_{\left.l^{\prime}\right\rfloor}\left(t^{\prime}\right)\right\rangle \\
\left\langle b_{l !}^{\dagger}(t) b_{l^{\prime} \uparrow}^{\dagger}\left(t^{\prime}\right)\right\rangle & \left\langle b_{l\rfloor}^{\dagger}(t) b_{\left.l^{\prime}\right\rfloor}\left(t^{\prime}\right)\right\rangle
\end{array}\right) .
\end{aligned}
$$

Detailed expressions of the self-energy and the spin Green's function are given in Appendix A.

It is important to note that the diagonal matrix elements of the spin propagator, $g^{11}\left(l, l^{\prime} ; t, t^{\prime}\right)$ and $g^{22}\left(l, l^{\prime} ; t, t^{\prime}\right)$ describe the "normal" propagation of the spin waves, i.e., propagation between sites of the same sublattice, while the off-diagonal matrix elements $g^{12}\left(l, l^{\prime} ; t, t^{\prime}\right)$ and $g^{21}\left(l, l^{\prime} ; t, t^{\prime}\right)$ describe the "abnormal" propagation of the spin waves, i.e., propagation between the two sublattices. This means that their values are nonzero only when $l$ and $l^{\prime}$ are on the same sublattice for the diagonal part or only when $l$ and $l^{\prime}$ belong to two different sublattices for the off-diagonal part, respectively. Put another way, the "normal" part is diagonal for the $S_{z}$ component, while the "obnormal" part describes 
the spin flip process. It is then clear from (19) that the Fock self-energy $\Sigma^{\mathrm{F}}\left(l, l^{\prime} ; t, t^{\prime}\right)$ will be zero when $l$ and $l^{\prime}$ belong to two different sublattices, which implies that with the Fock self-energy alone the hole can not move between the two sublattices. On the contrary, the Hartree self-energy $\Sigma^{\mathrm{H}}$ is nonzero when $l$ and $l^{\prime}$ are at nearest neighbour sites, belonging to two different sublattices so that the hole should be permitted to hop between sublattices. It has been pointed out in Ref. [10] that the Hartree term describing the spin background distortion gives rise to hole propagation over the entire lattice. The present formulation makes this point apparent and shows clearly that earlier treatments with Fock term alone $[8,9]$ can not describe hopping between sublattices.

In the presence of a hole, the spin background will be distorted. The distortion can be described by deviations of the order parameters (5) from their original values. The distorted values are:

$$
\begin{aligned}
& \Delta_{i j}=\left\langle\left(b_{i \uparrow}^{\dagger} b_{j \downarrow}^{\dagger}-b_{i \downarrow}^{\dagger} b_{j \uparrow}^{\dagger}\right) \exp \left(-i \int H_{\tau} d \tau\right)\right\rangle_{0}, \\
& \backslash_{i j}=\left\langle\left(b_{i \uparrow}^{\dagger} b_{j \uparrow}+b_{i \downarrow}^{\dagger} b_{j \downarrow}\right) \exp \left(-i \int H_{\tau} d \tau\right)\right\rangle_{0},
\end{aligned}
$$

where $H_{\tau}$ is the hopping hamiltonian (first term of $(4)$ in the interaction representation). Up to the first order, these order parameters turn out to be

$$
\begin{aligned}
\Delta_{i j}= & \Delta-t \sum_{\left\langle l^{\prime}\right\rangle} \int d \tau G\left(l, l^{\prime} ; \tau, \tau\right) \\
& {\left[g^{11}(i, l ; \tau) g^{21}\left(j, l^{\prime} ;-\tau\right)+g^{21}\left(i, l^{\prime} ; \tau\right) g^{22}(j, l ;-\tau)\right.} \\
& \left.-g^{11}(j, l ;-\tau) g^{21}\left(i, l^{\prime} ; \tau\right)-g^{21}\left(j, l^{\prime} ;-\tau\right) g^{22}(i, l ; \tau)\right], \\
\chi_{i j}= & -t \sum_{\left\langle l^{\prime}\right\rangle} \int d \tau\left(j\left(l, l^{\prime} ; \tau, \tau\right) .\right. \\
& {\left[g^{11}(i, l ; \tau) g^{11}\left(j, l^{\prime} ;-\tau\right)+g^{21}\left(i, l^{\prime} ; \tau\right) g^{12}(j, l ;-\tau)\right.} \\
& \left.+g^{12}(j, l ; \tau) g^{21}\left(i, l^{\prime} ;-\tau\right)+g^{22}\left(j, l^{\prime} ; \tau\right) g^{22}(i, l ;-\tau)\right] .
\end{aligned}
$$

As seen from the expressions of the self-energies (19) and the distorted order parameters (22), the self-energy $\Sigma^{\mathrm{H}}$ can not be expressed explicitly in terms of the order parameters $\Delta_{i j}$ and $\chi_{i j}$ in the Schwinger boson formalism. This is different from the standard spin wave representation adopted in Ref. [10], where the self-energy $\Sigma^{\mathrm{H}}$ could be expressed explicitly in terms of the spin distortion $\beta_{i}$. This difference is mainly due to the tact that the AF order parameter is defined on sites in the standard spin wave theory, while it is defined on bonds in the Schwinger boson formalism. However, the physics behind the expression of $\Sigma^{\mathrm{H}}$ should be the same, that is, the spin distortion, or the deviation of the order parameters $\Delta_{i j}$ and $\chi_{i j}$ due to the presence of a hole. This effect is rather similar to the multi-phonon processes appearing in the optical properties of the F-centres [14]. The BdeG equation (18) determines self-consistently the hole states and the spin distortion: The localized state is caused by the distortion which should be consistent with it at the same time.

So far we have discussed only hole states with a fixed origin. One can easily form Bloch states to recover the translational invariance. The Bloch state of a propagating hole can be defined as

$$
\left|\Psi_{k}\right\rangle=\left(P_{k} N\right)^{-1 / 2} \sum_{n} \exp \left(i \mathbf{k} \cdot \mathbf{R}_{n}\right)\left|\Psi_{0}\left(\mathbf{R}_{n}\right)\right\rangle
$$


where $\left|\Psi_{0}\left(\mathbf{R}_{n}\right)\right\rangle$ is the hole ground state in the Wannier representation. However, as defined by Eq.(15), it is a direct product of the hole part and the accompanying spin part, i.e., the hole propagates together with the distorted spin background. Here $P_{k}$ is the Fourier transform of the overlap integral $P_{n, 0}=\left\langle\Psi_{0}\left(\mathbf{R}_{n}\right) \mid \Psi_{0}(0)\right\rangle$ which is also a product of the hole and the spin parts. The spin overlap integral can be written as $e^{-S}$ with $S$ as the Huang-Rhys factor [14]. The energy of a propagating hole is then given by

$$
E_{k}=\left\langle\Psi_{k}|H| \Psi_{k}\right\rangle=P_{k}^{-1} \sum_{n} \exp \left(i \mathbf{k} \cdot \mathbf{R}_{n}\right) E_{n, 0},
$$

where $E_{n, 0}$ is the hopping matrix element between hole states localized at 0 and $\mathbf{R}_{n}$, respectively. Then the energy of a propagating hole depends on the overlap integral $P_{n, 0}$ and the hopping matrix element $E_{n, 0}$, which are given in Appendix B.

\section{RESULTS AND DISCUSSIONS}

First, by solving the quantum BdeG equations, we found a set of self-trapped states for a hole as in the previous works for the anisotropic model [10]. The lowest state is of $s$-symmetry, while the states immediately above it are doubly degenerate, of $p_{x}$ and $p_{y}$ symmetry, respectively. The higher states $d_{x y}$ and $d_{x^{2}-y^{2}}$ are not degenerate, but the energy difference is rather small. This result is also in agreement with the exact diagonalization on small clusters [7] and the self-consistent Born approximation [15,16] calculations, where more than one quasiparticle peaks are found in the spectral function. As compared with the previous calculations [10], we should mention two points here. One is that this calculation is for a single hole in an isotropic $2 \mathrm{~d}$ antiferromagnet, which is a better description of the high temperature oxide superconductors. Another point is that the self-trapped states survive even at finite temperatures where the AF order has become short-ranged.

Then within the quantum BdeG formalism, we have treated the local distortion of the spin configuration and the quantum renormalization process equally, i.e., both Hartree and Fock self-energy terms were included and treated at the same level for a fixed hole. With the Fock term alone (emission and reabsorption of spin waves), the hole moves only within one sublattice since the normal propagation of spin waves is within one sublattice while the abnormal propagation of spin waves is between two sublattices in an AF phase. The Hartree self-energy due to the spin background distortion makes the hole move over the whole lattice. On the other hand. for a moving hole, the Hartree self-energy vanishes due to the momentum conservation in the Born approximation. However, this is an artifact of the approximation involved, and one should resort to more sophisticated techniques, like instantons [17] to consider the hole hopping along with spin distortion.

To see the contributions of the Hartree and Fock self-energy terms separately, we show the wave functions of the ground state for a hole in Fig. 3 for $t / J=2$. With the Fock term alone, i.e., exactly at the level of the self-consistent Born approximation for a moving hole, the wave function in Fig. 3 (a) vanishes on one of the sublattices, i.e., the motion of the hole is restricted only to one sublattice. On the contrary, with the Hartree term alone, the wave function in Fig. 3 (b) seems to be more localized and does not vanish on the second sublattice due to the spin distortion effect. Of course, the Hartree term by itself will not do the job since the energies go like $-t^{2} / J \sim-U$, which is a wrong energy scale. It is necessary 
to include both the Hartree term and the Fock term, which reflects higher order virtual fluctuations and brings the energy scale back to $t$, and eventually to $J$. Fig. 3 (c) shows the wave function of the hole in the ground state obtained by including both the Hartree and Fock terms in the BdeG equation. Our calculations show that the shape of the wave function is not sensitive to the parameter $t / J$ within the range $(1 \div 4)$. The small size of the hole $(\approx 6$ lattice sites $)$ is the main reason why the hole self-trapped states survive in a short-ranged $\mathrm{AF}$ correlated state so long as the correlation length $(\xi \approx 25)$ is still much larger than the size of the hole state.

Moreover, we have studied the energy spectrum of the hole states as a function of the ratio $t / J$. The energy separation $\left(E_{2}-E_{1}\right)$ between the ground state $s$ and the second level $p_{x}$ or $p_{y}$ as a function of $t / J$ is plotted in Fig. 4 both for the case with the Fock term alone and with the Hartree term as well. The inclusion of the Hartree term, i.e., the distortion of the spin background due to the presence of the hole, leads to a reduction of the energy separation $E_{2}-E_{1}$, but it still remains finite. We expect the finite separation should correspond to a finite gap between the lowest two coherent peaks in the thermodynamic limit. This result was also obtained in the exact numerical calculation for a finite cluster [7].

Finally, by solving the BdeG equation, we could obtain the distorted AF order parameter $\Delta_{i j}$ and FM order parameter $\chi_{i j}$. which are shown in Table I, to the leading order. We see that the presence of a hole gives rise to FM correlations and reduces the AF correlation around the hole.

In summary, we have studied the problem of a single hole in an isotropic $2 \mathrm{~d}$ quantum antiferromagnet by using the quantum Bogoliubov-de Gennes method, which treats the distortion of the spin background due to the presence of the hole and the quantum fluctuations through the emission and reabsorption of virtual spin waves on an equal footing. We found there exists a series of self-trapped states for a hole, whose lowest state is of $s$-symmetry. The self-trapped hole states survive at finite temperatures when the AF order is short-ranged. By including the Hartree self-energy term we have shown that the hole can hop between the two sublattices. We also found that the relaxation of the spin background will reduce the separation between the two lowest self-trapped states $s$ and $p_{x}$ or $p_{y}$, which corresponds to a reduction of the energy gap between the two lowest coherent peaks.

\section{ACKNOWLEDGMENTS}

Part of this work was done when one of the authors (C.Q.W.) worked at ICTP as a research fellow. He is grateful to the Centre for its kind hospitality and its financial support.

\section{APPENDIX A: THE HOLE SELF-ENERGIES}

From Eq. (19), we can write down the retarded self-energy as

$$
\begin{aligned}
\Sigma_{r}^{\mathrm{H}}\left(\omega: l, l^{\prime}\right)= & \frac{1}{2} t^{2} I_{l l^{\prime}} \sum_{\left\langle m m^{\prime}\right\rangle} \int \frac{d \omega_{1}}{2 \pi} G_{c}\left(m, m^{\prime} ; \tau, \tau\right) . \\
& {\left[g_{r}^{22}\left(\omega_{1}, l-m\right) g_{c}^{11}\left(-\omega_{1}, l^{\prime}-m^{\prime}\right)\right.} \\
& +g_{c}^{22}\left(\omega_{1}, l-m\right) g_{r}^{11}\left(-\omega_{1}, l^{\prime}-m^{\prime}\right)
\end{aligned}
$$




$$
\begin{aligned}
& -g_{r}^{21}\left(\omega_{1}, l-m^{\prime}\right) g_{c}^{12}\left(-\omega_{1}, l^{\prime}-m\right) \\
& \left.-g_{c}^{21}\left(\omega_{1}, l-m^{\prime}\right) g_{r}^{12}\left(-\omega_{1}, l^{\prime}-m\right)\right]
\end{aligned}
$$

and

$$
\begin{aligned}
\Sigma_{r}^{\mathrm{F}}\left(\omega ; l, l^{\prime}\right)= & \frac{1}{2} t^{2} \sum_{m m^{\prime}} I_{l m} I_{l^{\prime} m^{\prime}} \int \frac{d \omega_{1} d \omega_{2}}{(2 \pi)^{2}} \\
& \left\{\left[g_{r}^{21}\left(\omega_{2}, l-m^{\prime}\right) g_{c}^{12}\left(\omega-\omega_{1}-\omega_{2}, m-l^{\prime}\right)\right.\right. \\
& +g_{c}^{21}\left(\omega_{2}, l-m^{\prime}\right) g_{r}^{12}\left(\omega-\omega_{1}-\omega_{2}, m-l^{\prime}\right) \\
& -g_{r}^{22}\left(\omega_{2}, l-l^{\prime}\right) g_{c}^{11}\left(\omega-\omega_{1}-\omega_{2}, m-m^{\prime}\right) \\
& \left.-g_{c}^{22}\left(\omega_{2}, l-l^{\prime}\right) g_{r}^{11}\left(\omega-\omega_{1}-\omega_{2}, m-m^{\prime}\right)\right] G_{c}\left(\omega_{1} ; m, m^{\prime}\right) \\
& +\left[g_{c}^{21}\left(\omega_{2}, l-m^{\prime}\right) g_{c}^{12}\left(\omega-\omega_{1}-\omega_{2}, m-l^{\prime}\right)\right. \\
& +g_{r}^{21}\left(\omega_{2}, l-m^{\prime}\right) g_{r}^{12}\left(\omega-\omega_{1}-\omega_{2}, m-l^{\prime}\right) \\
& -g_{c}^{22}\left(\omega_{2}, l-l^{\prime}\right) g_{c}^{11}\left(\omega-\omega_{1}-\omega_{2}, m-m^{\prime}\right) \\
& \left.\left.-g_{r}^{22}\left(\omega_{2}, l-l^{\prime}\right) g_{r}^{11}\left(\omega-\omega_{1}-\omega_{2}, m-m^{\prime}\right)\right] G_{r}\left(\omega_{1} ; m, m^{\prime}\right)\right\},
\end{aligned}
$$

where the subscripts $c$ and $r$ express the correlation and retarded Green's function, respectively.

In an $\mathrm{AF}$ background, we have the retarded spin Green's functions

$$
g_{r}(\omega, k)=\frac{1}{(\omega+i \eta)^{2}-E_{k}^{2}}\left(\begin{array}{cc}
\lambda+\omega & -\gamma_{k} \\
-\gamma_{k} & \lambda-\omega
\end{array}\right)
$$

and the correlation spin Green's function

$$
g_{c}(\omega, k)=-\frac{\pi i}{E_{k}} \operatorname{coth} \frac{\omega}{2 T}\left[\delta\left(\omega-E_{k}\right)-\delta\left(\omega+E_{k}\right)\right]\left(\begin{array}{cc}
\lambda+\omega & -\gamma_{k} \\
-\gamma_{k} & \lambda-\omega
\end{array}\right) .
$$

The retarded hole Green's function has been given in Eq.(16) and the correlation hole Green's function is

$$
G_{c}(\omega: i, j)=-2 \pi i \sum_{\alpha} \delta\left(\omega-E_{\alpha}\right)\left(1-2 N_{\alpha}\right) \psi_{\alpha}(i) \psi_{\alpha}^{\dagger}(j)
$$

where $N_{\alpha}$ is the hole occupation number of the $\alpha$-th energy level.

\section{APPENDIX B: THE OVERLAP INTEGRAL AND THE HOPPING MATRIX ELEMENT}

By solving the quantum BdeG equation (18), we can obtain self-consistently the excitation energies and the wave functions of the hole states with a fixed origin, and then the corresponding order parameters of the distorted spin background caused by the hole presence. Here we present the overlap integral $P_{n .0}$ and the hopping matrix element $E_{n, 0}$, both being direct products of the hole part and the corresponding spin background.

In terms of the distorted order parameters $\Delta_{i j}$ and $\chi_{i j}$, we can write down the Heisenberg Hamiltonian in the MF approximation as 


$$
H_{b}=H_{0}+H_{1},
$$

where $H_{0}$ is the MF Heisenberg Hamiltonian (15), while $H_{1}$ is the distortion induced by the hole

$$
H_{1}=J \sum_{k, k^{\prime}}\left[\delta \Delta_{k, k^{\prime}}\left(b_{k \uparrow} b_{-k^{\prime}+Q \downarrow}+b_{k \uparrow}^{\dagger} b_{-k^{\prime}+Q !}^{\dagger}\right)-\frac{1}{2} \chi_{k k^{\prime}}\left(b_{k \uparrow}^{\dagger} b_{k^{\prime} \uparrow}-b_{-k+Q \downarrow}^{\dagger} b_{-k^{\prime}+Q \downarrow}\right)\right] .
$$

Here we have carried out the Fourier transformation for the Schwinger bosons so that the order parameters in the above expression are given as follows

$$
\begin{aligned}
\delta \Delta_{k, k^{\prime}} & =\frac{1}{N} \sum_{i \in A} \sum_{\alpha}\left(\Delta_{i, i+\alpha}-\Delta\right) e^{-i\left(k-k^{\prime}\right) R_{\mathbf{t}}}\left(e^{i k_{\alpha}}+e^{-i k_{\alpha}^{\prime}}\right), \\
\chi_{k, k^{\prime}} & =\frac{1}{N} \sum_{i \in A} \sum_{\alpha} \chi_{i, i+\alpha} e^{-i\left(k-k^{\prime}\right) R_{i}}\left(e^{i k_{\alpha}}+e^{-i k_{\alpha}^{\prime}}\right) .
\end{aligned}
$$

Including the $H_{1}$ part, we have the new spin eigen-operators as

$$
\begin{aligned}
& \tilde{\alpha}_{k}=\alpha_{k}+\sum_{k^{\prime}} \Lambda_{k, k^{\prime}} \beta_{k^{\prime}}, \\
& \dot{\beta}_{k}=\beta_{k}+\sum_{k^{\prime}} \Lambda_{k, k^{\prime}} \alpha_{k^{\prime}},
\end{aligned}
$$

where $\Lambda_{k, k^{\prime}}$ are determined by the distorted order parameters. Then we have the distorted spin background

$$
|G\rangle=\exp \left(\sum_{k, k^{\prime}} \Lambda_{k, k^{\prime}} \alpha_{k}^{\dagger} \beta_{k^{\prime}}^{\dagger}\right)|g\rangle,
$$

with $|g\rangle$ as the spin background without distortion. Now we can perform the calculations of the overlap integrals and hopping matrix elements between the hole states at different origins. The calculation is quite lengthy, so we quote here only the final results:

1. The overlap integral

$$
\left\langle G^{\prime} \mid G\right\rangle=\exp \{-\operatorname{Tr} \ln (1-C)\}
$$

where the matrix $C$ has its elements as follows

$$
C_{k, k^{\prime}} \equiv \sum_{k^{\prime \prime}} \frac{1}{1-e^{-\beta E_{k}}} \Lambda_{k, k^{\prime \prime}}^{\prime} \frac{1}{1-e^{-\beta E_{k^{\prime \prime}}}} \Lambda_{k^{\prime \prime} \cdot k^{\prime}}
$$

2. The hopping matrix element

$$
\begin{array}{r}
\left\langle G^{\prime}\left|b_{k \uparrow}^{\dagger} b_{k^{\prime} \uparrow}\right| G\right\rangle=\left\langle G^{\prime} \mid G^{\prime}\right\rangle\left\{u_{k} u_{k^{\prime}}\left[(1-A)^{-1}-1\right]_{k, k^{\prime}}+u_{k^{\prime}} v_{k^{\prime}}\left[(1-A)^{-1} \Lambda^{\prime}\right]_{k, k^{\prime}}\right. \\
\left.+v_{k} u_{k^{\prime}}\left[\Lambda^{\mathrm{T}}(1-A)^{-1}\right]_{k, k^{\prime}}+v_{k} v_{k^{\prime}}\left[(1-B)^{-1}\right]_{k, k^{\prime}}\right\},
\end{array}
$$

where $u_{k}$ and $v_{k}$ are transformation functions shown in Eq.(9), and the matrix elements

$$
\begin{aligned}
& A_{k, k^{\prime}}=\sum_{m} \frac{1}{1-e^{-\beta E_{k}}} \Lambda_{k, m}^{\prime} \frac{1}{1-e^{-\beta E_{m}}} \Lambda_{k^{\prime}, m}, \\
& B_{k, k^{\prime}}=\sum_{m} \frac{1}{1-e^{-\beta E_{m}}} \Lambda_{m, k}^{\prime} \frac{1}{1-e^{-\beta E_{k}}} \Lambda_{m, k^{\prime}} .
\end{aligned}
$$

The calculation for the hole part is straightforward, so the overlap integrals $P_{n, 0}$ and the hopping matrix elements $E_{n, 0}$ between the hole states with different origins can be obtained easily. 


\section{REFERENCES}

[1] J.G. Bednorz and K.A. Müller, Z. Phys. B64, 188(1986); C.W. Chu et al., Phys. Rev. Lett. 58, 405(1987).

[2] R.J. Birgeneau and G. Shirane, in Physics Properties of High Temperature Superconductors, Vol. 1, edited by D.M. Ginsberg (World Scientific, Singapore, 1989)p.151; J.P. Rossat-Mignod, L.P. Regnault, J.M. Jarguens, P. Burlet, J.Y. Henry, and G. Lapertot, in Dynamics of Magnetic Fluctuations in High Temperature Superconductors, G. Reiter, P. Horsch, and G.C. Psaltakis, eds. (Plenum, New York, 1991) p. 35.

[3] P.W. Anderson, Science 235, 1196(1987); in Frontiers and Borderlines in Many-Body Physics, J.R. Schrieffer and R.A. Broglia, eds. (North-Holland, Amsterdam, 1987), p.1.

[4] See, e.g., K.A. Chao et al., J. Phys. C 10, L271(1977); J.E. Hirsch, Phys. Rev. Lett. 54, 1371(1.985); A.H. MacDonald, S.M. Girvin, and D. Yoshioka, Phys. Rev. B 37, $9753(1988)$.

[5] F.C. Zhang and T.M. Rice, Phys. Rev. B 37, 3759(1988).

[6] L. Yu, Z.B. Su, and Y.M. Li, Chin. J. of Phys. 31, 579 (1993).

[7] D. Poilblanc, H.J. Schulz, and T. Ziman, Phys. Rev. B 46, 6435(1992); ibid, 47, 3268(1993); D. Poilblanc, 'T. Ziman, H.J. Schulz, and E. Dagotto, ibid, 47, 14267(1993).

[8] S. Schmitt-Rink, C.M. Varma, and A.E. Ruckenstein, Phys. Rev. Lett. 60, 2793(1988).

[9] C.L. Kane, P.A. Lee, and N. Read, Phys. Rev. B 39, 6880(1989).

[10] Z.B. Su, Y.M. Li, W.Y. Lai, and L. Yu, Phys. Rev. Lett. 63, 1318(1989); Int. J. Mod. Phys. B3, 1913( 989); Y.M. Li, W.Y. Lai, Z.B. Su, and L. Yu, Phys. Rev. B 47, $7938(1993)$.

[11] See, e.g., Y. Toyozawa and Y. Shinozuka, J. Phys. Soc. Jpn 48, 472 (1980)

[12] D. Arovas and A. Auerbach. Phys. Rev. B 38, 316(1988); A. Auerbach and D. Arovas, Phys. Rev. Lett. 61, 617(1988).

[13] D. Yoshioka, J. Phys. Soc. Jpn. 58, 32(1989); 58, 1516(1989): in Strong Correlation and Superconductivity, edited by H. Fukuyama et al.(Springer, Heidelberg, 1989), p.124.

[14] K. Huang and A. Rhys, Proc. Roy. Soc. A204, 406(1950).

[15] G. Martinez and P. Horsch, Phys. Rev. B 44, 317(1991).

[16] Z. Liu and E. Manousakis, Phys. Rev. B 44, 2414(1991); ibid, 45, 2425(1992).

[17] See, e.g., K. Yonemitsu, Submitted to Phys. Rev. B. 


\section{TABLES}

TABLE I. The AF order parameter $\Delta_{i, j}=\Delta+\delta \Delta_{i j}$ and the FM order parameter $\chi_{i j}$ in the distorted background, where $\Delta=0.579$, site $i$ is on sublattice $A, j$ is its nearest neighbour site and they are labeled as in Fig. 5.

\begin{tabular}{||c||c|c|c|c|c|c|c|c|c|c|c||}
\hline \hline$(i, j)$ & $(0,1)$ & $(2,1)$ & $(3,1)$ & $(2,4)$ & $(3,4)$ & $(5,4)$ & $(3,6)$ & $(7,4)$ & $(7,6)$ & $(5,8)$ & $(7,8)$ \\
\hline$\delta \Delta_{i j}$ & -0.13 & -0.06 & -0.04 & -0.02 & -0.01 & -0.01 & 0.00 & 0.00 & 0.00 & 0.00 & $\mathbf{0 . 0 0}$ \\
\hline$\chi_{i j}$ & 0.29 & 0.20 & 0.14 & 0.10 & 0.06 & 0.03 & 0.03 & 0.02 & 0.01 & 0.01 & 0.00 \\
\hline \hline
\end{tabular}




\section{FIGURES}

FIG. 1. The density of spin-wave excitations $\zeta(E)$ (solid line) as defined in the text and the density of spin-wave states $\rho(E)$ (dashed line) at a finite temperature $T / \lambda=0.38$, where the spin-wave gap is $E_{g} / \lambda=0.01$.

FIG. 2. The self-energy diagrams for a hole: (a) Hartree and (b) Fock terms. The solid lines are for holes and the dashed lines are for spins.

FIG. 3. The wave functions of the ground state for the hole at $t / J=2$ obtained by solving the BdeG equation: (a) with Fock term alone, (b) with Hartree term alone and (c) with both Hartree and Fock terms.

FIG. 4. The energy separation between the two lowest hole states as a function of $t / J$ obtained by solving the BdeG equation with Fock term alone (dashed line) and with both Hartree and Fock terms (solid line).

FIG. 5. The site labels. The center of the hole is at site 0 . 


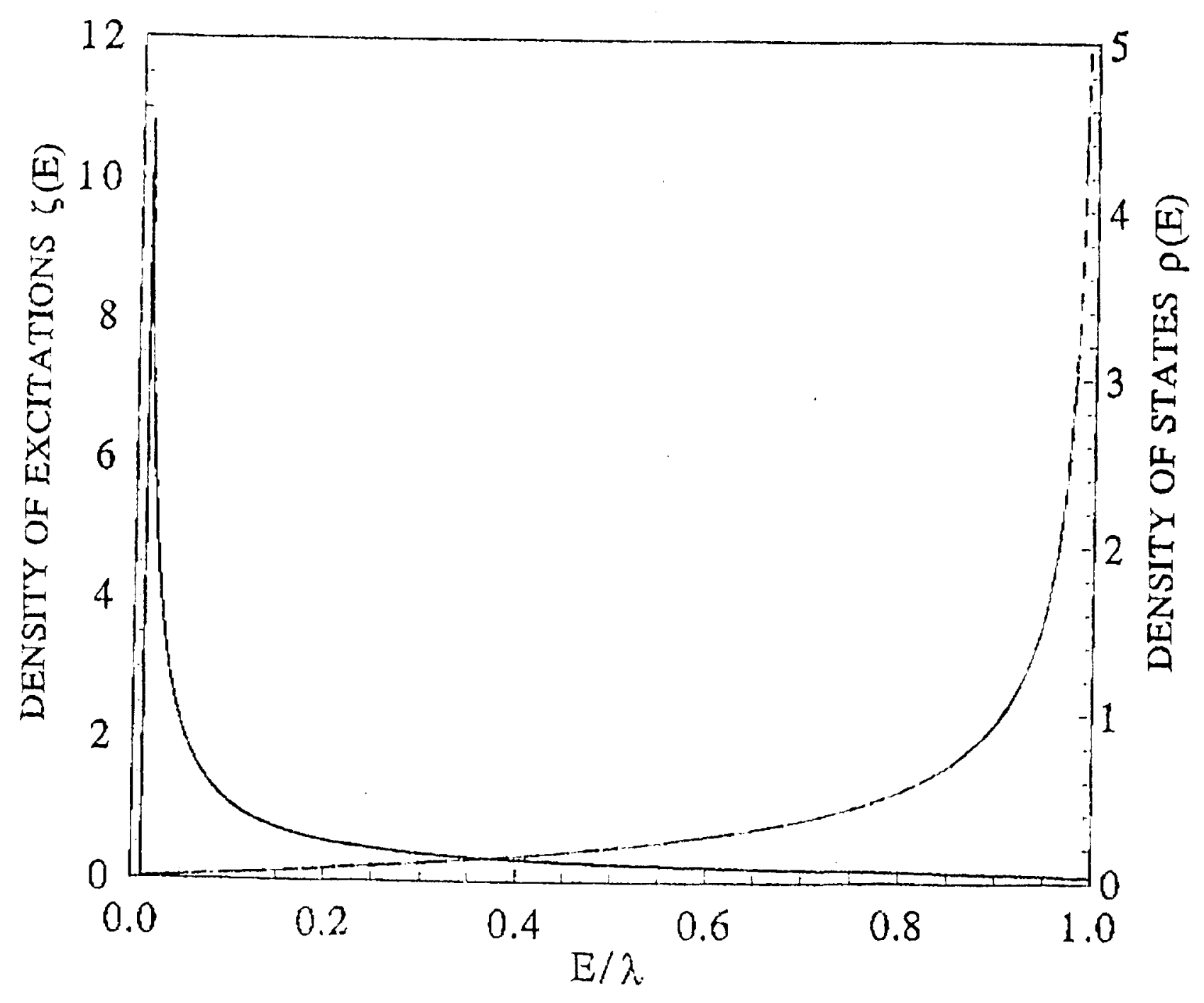

Fig. 1

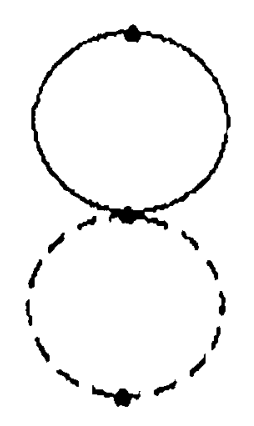

(a)

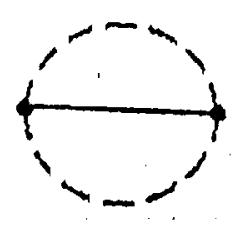

(b)

Fig.2 


$$
\begin{aligned}
& 0.0200 .0 \\
& 0.00 \quad 0.00 \quad 0.00 \\
& \begin{array}{lllll}
0.03 & 0 & 0.13 & 0 & 0.03
\end{array} \\
& \begin{array}{lllll}
0.00 & 0.00 & 0.00 & 0.00 & 0.00
\end{array} \\
& \begin{array}{lllllll}
0.02 & 0 & 0.29 & 0 & 0.29 & 0 & 0.02
\end{array} \\
& \begin{array}{lllllll}
0.00 & 0.00 & 0.01 & 0.35 & 0.01 & 0.00 & 0.00
\end{array} \\
& \begin{array}{lllllll}
0 & 0.13 & 0 & 0.77 & 0 & 0.13 & 0
\end{array} \\
& \begin{array}{lllllll}
0.00 & 0.00 & 0.35 & 0.71 & 0.35 & 0.00 & 0.00
\end{array} \\
& \begin{array}{lllllll}
0.02 & 0 & 0.29 & 0 & 0.29 & 0 & 0.02
\end{array} \\
& \begin{array}{lllllll}
0.00 & 0.00 & 0.01 & 0.35 & 0.01 & 0.00 & 0.00
\end{array} \\
& \begin{array}{lllll}
0.03 & 0 & 0.13 & 0 & 0.03
\end{array} \\
& \begin{array}{lllll}
0.00 & 0.00 & 0.00 & 0.00 & 0.00
\end{array} \\
& \begin{array}{lll}
0.02 & 0 & 0.02
\end{array} \\
& \begin{array}{lll}
0.00 & 0.00 & 0.00
\end{array}
\end{aligned}
$$

(a)

(b)

\author{
$\begin{array}{lll}0.05 & 0.06 & 0.05\end{array}$ \\ $\begin{array}{lllll}0.08 & 0.13 & 0.16 & 0.13 & 0.08\end{array}$

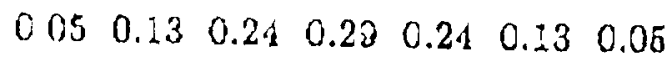 \\ $\begin{array}{lllllll}0.06 & 0.16 & 0.28 & 0.38 & 0.29 & 0.16 & 0.06\end{array}$ \\ $\begin{array}{lllllll}0.05 & 0.13 & 0.24 & 0.20 & 0.24 & 0.13 & 0.05\end{array}$ \\ $\begin{array}{lllll}0.08 & 0.13 & 0.16 & 0.13 & 0.08\end{array}$ \\ $\begin{array}{lll}0.05 & 0.06 & 0.05\end{array}$
}

(c)

Fig.3 


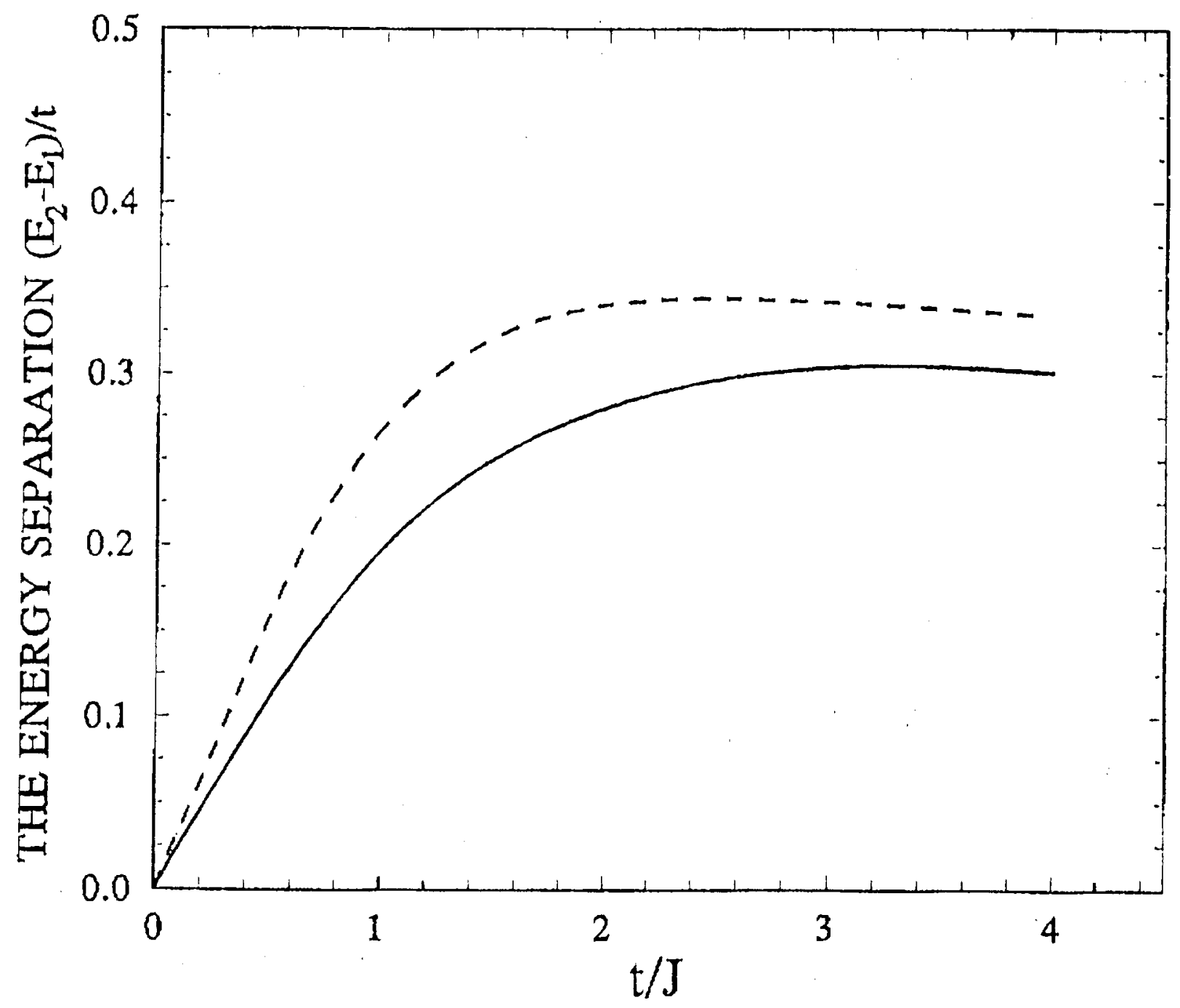

Fig. 4

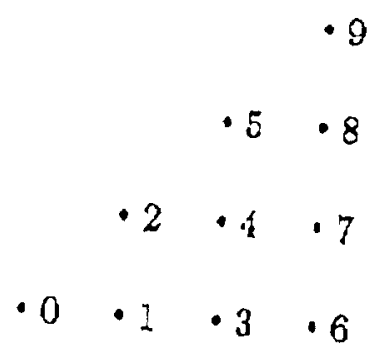

Fig. 5 
